might tell others. Some were wary of disclosing to female nurses from their community. It is also difficult for men to reveal their HIV-positive status to their partners fearing that they will be viewed as a cheater or someone with a promiscuous past. Knowing that the person disclosed to is also HIV positive makes it easier for the men in our study to disclose their HIV status.

Conclusion Our findings show that men often weigh up the costs and benefits of disclosing their HIV-positive status depending on who they are disclosing to. Further research is needed to understand different ways in which men can disclose their HIV-positive status without fear of stigmatization.

\section{P145 A QUALITATIVE STUDY ON SEXUALISED DRUG USE AMONG MALE AND TRANSGENDER WOMEN SEX WORKERS IN AMSTERDAM, THE NETHERLANDS}

\begin{abstract}
${ }^{1} \mathrm{~S}$ Druckler*, ${ }^{1} \mathrm{H}$ Zimmermann, ${ }^{1} \mathrm{~S}$ Eekman, ${ }^{1} \mathrm{M}$ van Rooijen, ${ }^{1,2} \mathrm{U}$ Davidovich, ${ }^{1,3} \mathrm{H}$ de Vries, ${ }^{1} \mathrm{~T}$ Heijman. 'Public Health Service of Amsterdam, Department of Infectious Diseases, Amsterdam, The Netherlands; 'Department of Social Psychology, University of Amsterdam, Amsterdam, The Netherlands; ${ }^{3}$ Department of Dermatology, Amsterdam Institute for Infection and Immunity (AlandII), Amsterdam University Medical Centers, Amsterdam, The Netherlands
\end{abstract}

\subsection{6/sextrans-2021-sti.254}

Background The objective of this study was to provide insight into the motives of drug use during work among male sex workers (MSW) and transgender women sex workers (TSW) and its possible effect on sexual behaviour that may increase the chance of STIs and/or HIV acquisition.

Methods From March to June 2018, we conducted semi-structured in-depth interviews among MSW and TSW visiting the Prostitution and Health Center in Amsterdam. Transcribed interviews were analysed by two researchers with an opencoding process, after which similar codes were categorized into themes.

Results We conducted 23 interviews among 15 MSW and 8 TSW. Almost all sex workers $(n=21)$ reported any sexualised drug use (irrespectively of work or private situation) and the majority $(n=14)$ reported illicit drug use during work. The initiative of using drugs most often comes from the clients.

Motives for using or not using drugs during work were categorized into self-oriented and client-oriented motives. Selforiented motives to use drugs included receiving more money, increasing pleasure during sex and the ability to work more hours. Self-oriented motives for NOT using drugs included to keep track of time, to control safe sex and to maintain professional distance. Client-oriented motives to use drugs included client asks for it and to make client happy. Clientoriented motives to NOT use drugs included preserving sexual functioning.

Participants reported that due to drug use they forget/stop/ skip using condoms during oral or anal sex. A self-applied prevention strategy that was described was staying away from actual sex acts when using drugs because the judgment over condom use could get influenced.

Conclusions Future prevention should take into account that the initiative of using drugs most often comes from the clients and provides work-related added value. Assertively training and harm-reduction strategies described by the sex workers can be used by developing interventions.

\section{P147 FACTORS RELATED TO HEPATITIS C VIRUS (HCV) TESTING AMONG PERSONS WHO INJECT DRUGS IN THE MEMPHIS METROPOLITAN AREA}

1J Marr*, 'L Sizemore, ${ }^{2} \mathrm{~L}$ Pichon, ${ }^{1} \mathrm{R}$ Garman, ${ }^{1} \mathrm{~S}$ Mathieson, ${ }^{1} \mathrm{M}$ Brantley. ${ }^{1}$ Tennessee Department of Health, Nashville, US; ${ }^{2}$ University of Memphis School of Public Health, Division of Social and Behavioral Sciences, Memphis, US

\subsection{6/sextrans-2021-sti.255}

Background Hepatitis C virus (HCV) infection is both underdiagnosed and underreported. Every three years, the National HIV Behavioral Surveillance (NHBS) project, led by the Tennessee Department of Health (TDH) monitors HIV and HCV risk behaviors and outcomes among people who inject drugs (PWID) in the Memphis metropolitan area. The objective of this study was to understand characteristics associated with HCV testing history.

Methods During August-December 2018, TDH interviewed residents from the Memphis metropolitan area who selfreported injection drug use in the past 12 months utilizing respondent-driven sampling. We assessed independent correlation of self-reported HCV testing history with various demographic and risk behaviors using Chi-square tests. Multivariate logistic regression was performed on covariates with statistically significant differences $(\mathrm{p}<0.05)$ to determine odds ratios (OR) and 95\% confidence intervals (CI).

Results Among 530 participants, daily injection was common $(\mathrm{N}=439,83 \%)$. Approximately half $(\mathrm{N}=297,56 \%)$ reported ever being tested for HCV. Non-Hispanic white race/ethnicity, history of overdose, polydrug use (reporting multiple drug types), long-term drug use (first injecting drugs $\geq 15$ years prior), and visiting a healthcare provider within the past 12 months were independently associated with HCV testing $(\mathrm{p}<0.05)$. HCV testing was more likely in participants who had visited a healthcare provider within the past 12 months (OR: 2.3, 95\% CI: 1.6-3.4), were non-Hispanic white (OR: 2.2, 95\% CI: 1.5-3.3), reported long-term drug use (OR: 1.7, 95\% CI: 1.1-2.7), and polydrug use (OR: 1.7, 95\% CI: $1.0-$ 3.9).

Conclusion Nearly half of participants in the 2018 NHBS IDU cycle had never been tested for HCV despite ongoing high-risk behaviors. Participants who reported long-term drug use or visiting a healthcare provider within the past 12 months were more likely to report HCV testing. These findings underscore the need to integrate $\mathrm{HCV}$ testing into and improve access to routine care for PWID in the Memphis area.

\section{P148 PREVALENCE AND CORRELATES OF SEXTING AND CYBERSEX USE AMONG ADULTS BEFORE AND DURING COVID-19 SOCIAL DISTANCING MEASURES IN PANAMA}

${ }^{1,2} \mathrm{~A}$ Gabster* ${ }^{3}$ J Toller Erausquin, ${ }^{1} \mathrm{P}$ Mayaud, ${ }^{4} \mathrm{~K}$ Michielsen, ${ }^{2} \mathrm{~J}$ Pascale, ${ }^{4} \mathrm{C}$ Pericas Escalé,
${ }^{1} \mathrm{M}$ Marks, ${ }^{2} \mathrm{~J}$ Katz, ${ }^{2} \mathrm{M}$ de Argote, ${ }^{2} \mathrm{G}$ Cabezas Talavero, ${ }^{2} \mathrm{~A}$ Murillo Estrada, ${ }^{1,5} \mathrm{~J}$ Tucker.
${ }^{1}$ London School of Hygiene and Tropical Medicine, London, UK; ${ }^{2}$ Instituto Conmemorativo
Gorgas De Estudios De La Salud, Panama, Panama; ${ }^{3}$ University of North Carolina
Greensboro, USA, School of Health and Human Sciences, Greensboro, USA; ${ }^{4}$ Universiteit
Gent, Belgium, Faculty of Medicine and Health Sciences, Gent, Belgium; ${ }^{5}$ University of North
Carolina at Chapel Hill, Institute for Global Health and Infectious Diseases, Chapel Hill, USA

10.1136/sextrans-2021-sti.256

Background Lockdown and other COVID-19 social distancing measures (COVID-19-measures) may influence virtual sex 
behaviour due to increased screen time and decreased in-person sexual activity. This analysis describes the prevalence and factors associated with virtual sex before and during COVID19 measures in Panama.

Methods An online survey conducted among $\geq 18$ years individuals residing in Panama using social media recruitment, from August 8-September 12, 2020, at the end of strict lockdown measures. Questions included demographics, virtual sex (sexting [sharing/receiving nude/semi-nude photos/video] and cybersex [sexual acts in front of a camera] three months before and during COVID-19-measures. Logistic regression was used to identify associations with increased use of virtual sex.

Results Overall, 960 individuals participated; 526 (54.8\%) identified as cis-women, $366(31.1 \%)$ as cis-men, and 68 $(7.1 \%)$ as non-binary/another gender; median age was $28 \mathrm{y}$ (IQR:23-37y). Before COVID-19-measures, 44.1\% (369/837) reported sexting, 20.4\% (172/842) cybersex, 46.4\% (392/485) virtual sex. During COVID-19-measures, sexting, cybersex and virtual sex increased for 17.4\%(139/797), 9.4\%(74/790), and $19.9 \%(159 / 800)$ of participants, respectively. More cis-men reported virtual sex increase than cis-women $(25.7 \%$ vs $17.2 \%$ [rural/urban adjusted]AOR=1.69, 95\%CI:1.18-2.43). Bisexual $(38.7 \%$ [gender and urban/rural adjusted]AOR $=2.08,95 \%$ CI:1.09-3.95) and lesbian/gay participants $(42.4 \%, \mathrm{AOR}=2.64$, 95\%CI:1.47-4.73) reported virtual sex increase more frequently compared to heterosexual participants (16.1\%). Increase in casual sex was associated with increase in virtual sex $(45.0 \%$ vs less casual sex 25.3\%, AOR=4.06, 95\% CI:1.24-13.35). Increased pornography use was associated with increased virtual sex $(52.0 \%$ vs $7.8 \%$ decreased pornography, $\mathrm{AOR}=5.68,95 \% \mathrm{CI}: 2.40-13.44)$. Among participants with a long-term partner, virtual sex increased among those who reported more partnership conflicts during than before COVID-19-measures $(27.8 \%$ vs $12.8 \%$ among those who reported fewer conflicts, $\mathrm{AOR}=2.88,95 \% \mathrm{CI}: 1.45-5.72$ ).

Conclusions Virtual sex was common before COVID-19-measures in Panama. During COVID-19-measures, virtual sex increased among cis-men, lesbian/gay and bisexual participants. Virtual sex was associated with increased pornography use, casual sex, and increased conflicts with long-term partners.

\section{P149 IMPLEMENTING TARGETED MOLECULAR RESISTANCE TESTING FOR GONORRHOEA IN A LARGE URBAN UK CLINIC}

${ }^{1} E$ Moss, ${ }^{1} A$ Maxwell, ${ }^{2} E$ Goldstein, ${ }^{2} \mathrm{R}$ Gunson, 'A Winter. 'Sandyford Sexual Health Service, NHS Greater Glasgow and Clyde, Glasgow, UK; ${ }^{2}$ West of Scotland Specialist Virology Centre, Glasgow, UK

10.1136/sextrans-2021-sti.257

Background Sandyford Sexual Health serves a population of ca. 1 million residents of Greater Glasgow and Clyde, UK. We use RealTime CT/NG assay (Abbott Molecular) to exclude gonorrhoea, all positive results are centrally notified and managed. We report our experience implementing 'resistanceguided' treatment for gonorrhoea using the SpeeDx ResistancePlus ${ }^{\circledR}$ GC test. The objective was to measure the proportion of patients successfully managed with oral rather than injectable treatment.
Methods Resistance testing was targeted at patients who might benefit, excluding patients who had empirical treatment. This was determined by our laboratory scientist who reviewed the electronic prescribing record. Tests were run twice weekly. Meaningful test name and results messages were designed to address anticipated human factors in assimilating the information.

Results Between 16th October and 24th December 2020, 93 patients had a positive gonorrhoea test meriting treatment, of which $20(22 \%)$ had infection at more than one anatomical site. Empirical treatment was given to 36 patients and so did not undergo resistance testing; 3 had insufficient residual sample. In the remaining 54 patients, 3 were gonorrhoea negative via SpeeDx ResistancePlus ${ }^{\circledR}$ testing and 2 were gyrA indeterminate. Ultimately, 33 had predicted ciprofloxacin sensitivity; 16 had predicted ciprofloxacin resistance.

Of the 33 patients identified as ciprofloxacin sensitive, 16 received oral ciprofloxacin and 17 received alternative standard-of-care. Reasons for not using ciprofloxacin included: clinician preference for injectable, resistance results not being available in time and quinolone contraindication.

No patients had discordant resistance predictions at different anatomical sites.

Conclusions Targeted molecular resistance testing for gonorrhoea averted injectable treatment in $17 \%$ of this cohort with around only half being eligible for testing due to immediate or prior treatment. We also averted some injections amongst contacts. Future work will focus on building clinician confidence and improving turnaround time in a production environment.

\section{P150 CHARACTERIZATION OF PHARYNGEAL GONORRHEA IN UGANDAN MEN WITH URETHRAL DISCHARGE SYNDROME}

${ }^{1} \mathrm{~J}$ Melendez ${ }^{*},{ }^{2} \mathrm{E}$ Mande, ${ }^{2} \mathrm{~A}$ Onzia, ${ }^{3} \mathrm{P}$ Kyambadde, ${ }^{2} \mathrm{M}$ Lamorde, ${ }^{1} \mathrm{Y}$ Manabe, ${ }^{1} \mathrm{M}$ Hamill. ${ }^{1} J o h n s$ Hopkins School of Medicine, Baltimore, USA; ${ }^{2}$ Infectious Diseases Institute, Makerere University, Kampala, Uganda; ${ }^{3}$ Ministry of Health, STI Control Unit, Kampala, Uganda

\subsection{6/sextrans-2021-sti.258}

Background Multi-drug resistant Neisseria gonorrhoeae (NG) threatens the effectiveness of gonorrhea treatment and control globally. Pharyngeal NG (pNG) infections are hypothesized to play a role in the emergence of antimicrobial resistance (AMR) in NG, yet the epidemiology of pNG infections is poorly understood, especially in resource-limited settings (RLS). We report on pNG in men with concurrent urogenital NG infections in Kampala, Uganda.

Methods Penile-meatal and pharyngeal samples were collected from Ugandan men with urethral discharge syndrome (UDS), cultured for NG, and tested by nucleic acid amplification tests (NAATs) for NG and other STIs. Antimicrobial susceptibility testing was performed on all NG isolates.

Results Of the 83 participants (mean age 29.5 years) who provided urogenital and pharyngeal samples, 22.9\% (19/83) were living with HIV, and $57.8 \%$ (48/83) were positive for urogenital gonorrhea. NG was detected in $8.6 \%$ (7/81) of pharyngeal samples by NAAT; $57.1 \%$ (4/7) were also positive by culture. Participants with pNG had concurrent urogenital NG, were HIV-negative, heterosexual (71.4\%), reported transactional sex 